\title{
Hypoxemia in Pulmonary Embolism, a Clinical Study
}

\author{
James E. Wilson III, Alan K. Pierce, Robert L. Johnson, Jr., \\ Edward R. Winga, W. Ross Harrell, George C. Curry, and \\ Charles B. Mullins \\ From the Pauline and Adolph Weinberger Laboratory for Cardiopulmonary \\ Research, Department of Internal Medicine, University of Texas (Southwestern) \\ Medical School at Dallas, Dallas, Texas 75235
}

A B S T R A C T The cause of hypoxemia was studied in 21 patients with no previous heart or lung disease shortly after an episode of acute pulmonary embolism. The diagnosis was based on pulmonary angiography demonstrating distinct vascular filling defects or "cutoffs." It was found that virtually all of the hypoxemia in patients with previously normal heart and lungs could be accounted for on the basis of shunt-like effect. The magnitude of the shunting did not correlate with the percent of the pulmonary vascular bed occluded nor with the mean pulmonary artery pressure. The shunts tended to gradually recede over about a month after embolism. Patients without pulmonary infarction were able to inspire $80-111 \%$ of their predicted inspiratory capacities, and this maneuver temporarily diminished the observed shunt. Patients with pulmonary infarcts were able to inhale only to 60 $69 \%$ of predicted inspiratory capacity, and this did not reverse shunting. These data suggest that the cause of right-to-left shunting in patients with pulmonary emboli is predominantly atelectasis.

When the elevation of mean pulmonary artery pressure was compared to cardiac index per unit of unoccluded lung, it fell within the range of pulmonary hypertension predicted from published data obtained in patients with exercise in all except one case. This observation suggests that pulmonary vasoconstriction following embolism is not important in humans, although these data are applicable only during the time interval in which our patients were studied and in patients receiving heparin.

This study was presented in part at the 1969 meeting of the American Society for Clinical Investigation.

Dr. Wilson was a U. S. Public Health Service Special Research Fellow (HE 35, 818). Dr. Winga was a Trainee supported by U. S. Public Health Service Training Grant HE 05812.

Received for publication 17 June 1970 and in revised form 16 November 1970.

\section{INTRODUCTION}

Hypoxemia usually accompanies pulmonary embolism in man (1), but its causes are ill understood, and its natural course is poorly documented. Attempts to determine the cause of the hypoxemia have been made in laboratory animals, but results are contradictory. Moreover, animal studies may not be satisfactory for describing the physiologic events after pulmonary embolism in man for the following reasons. (a) Since animals must be anesthetized, they are studied either under conditions of blunted respiratory response or artificial ventilation. (b) Animals usually are studied immediately after embolization without extended observations. (c) The response to foreign body or autologous blood clot emboli may differ from the response to emboli that have existed as thrombi in peripheral veins. $(d)$ There are considerable species differences in the responses to emboli (2-5).

Interpretation of previous studies in man concerning the cause of hypoxemia after pulmonary embolism is impaired by the following factors. (a) The interval from the first symptom of embolization to the study is frequently over a month $(6-8)$. (b) Patients may have congestive heart failure (9-11) or unrelated pulmonary disease (12) as a cause for hypoxemia. (c) Patients with recurrent emboli are not separated from those with a single episode of embolization (7). (d) Postoperative patients, who may have other causes for hypoxemia, are included (13). (e) The diagnosis frequently is made only on clinical grounds, and the extent of embolic occlusion is seldom assessed. $(f)$ Serial studies on the same patients are not available.

The purposes of the present study were to determine in patients without other heart or lung disease the mechanisms and course of the hypoxemia associated with proven pulmonary emboli and the relationship of hypoxemia to the extent of embolic occlusion. 


\section{METHODS}

Study plan. Patients had a right heart catheterization in the supine position as soon as possible after pulmonary embolism was suspected. Cardio-green dye was injected from the superior vena cava and pulmonary artery in order to estimate cardiac output and to detect intracardiac rightto-left shunts. Pressures were recorded in the right atrium, ventricle, and pulmonary artery; wedge pressures were obtained when possible. Expired gas was collected for measurements of oxygen consumption and carbon dioxide production while the patient breathed room air; simultaneously, samples of arterial and mixed venous blood were collected for measurement of $\mathrm{pH}$ and blood gases. Blood collection was repeated after breathing $100 \%$ oxygen for $15 \mathrm{~min}$ in order to estimate venous admixture due to true right-to-left shunting. Next, pulmonary angiograms were obtained through a No. 8 catheter with the tip in the main pulmonary artery, injecting $1 \mathrm{mg}$ per $\mathrm{kg}$ of Renografin $76^{1}$ up to a maximum of $70 \mathrm{ml}$.

On one to three occasions during the next $4 \mathrm{wk}$, expired gas was collected and arterial blood gases were measured while the patient breathed room air and then $100 \%$ oxygen in the supine position for $15 \mathrm{~min}$. During $100 \%$ oxygen breathing, completeness of nitrogen $\left(\mathrm{N}_{2}\right)$ wash out was determined by continuously monitoring the exhalate with a nitrogen analyzer. The effect of deep breathing (voluntary or induced by intermittent positive pressure at $20-40 \mathrm{~cm}$ $\left.\mathrm{H}_{2} \mathrm{O}\right)$ on the arterial oxygen tension $\left(\mathrm{PaO}_{2}\right)$ was noted; the maximum tidal volume during deep breathing was recorded. Spirometry was performed serially after each patient was free of pleuritic chest pain. Eight patients had repeat right heart catheterizations and pulmonary angiograms 13-35 (average 22) days after the first study. An additional patient had repeat angiograms at 3 and 13 months.

To further elucidate the cause of hypoxemia, the following studies were performed in selected patients: (a) slow space determination by Briscoe's two balloon-collecting system measuring expired nitrogen concentration at 1 -min intervals while breathing $100 \%$ oxygen $(14-16)$; $(b)$ airway resistance by the method of DuBois, Botelho, and Comroe
(17); and (c) dynamic compliance by the method of von Neergaard, and Wirz (18).

Analytical methods. Blood oxygen saturation was measured with a reflection oximeter, and blood $\mathrm{pH}$, carbon dioxide tension $\left(\mathrm{PaCO}_{2}\right)$, and $\mathrm{PaO}_{2}$ were measured, respectively, by a glass $\mathrm{pH}$ electrode, Severinghaus $\mathrm{P}_{\mathrm{co}_{2}}$ electrode, and modified Clark $\mathrm{P}_{\mathrm{O}_{2}}$ electrode. Each instrument was calibrated just prior to each analysis with standard gases of known composition. Expired gas analysis was performed by infrared and paramagnetic gas analyzers or Scholander microanalysis. These methods have equal accuracy in our laboratory (19). Volumes were determined with a Tissot spirometer.

Blood obtained while the patient breathed $100 \%$ oxygen was injected into the cuvette of the $\mathrm{P}_{\mathrm{O}_{2}}$ electrode within 2 min after withdrawal from the patient. When the $\mathrm{Pa}_{2}$ was $300-600 \mathrm{~mm} \mathrm{Hg}$ the $\mathrm{P}_{\mathrm{O}_{2}}$ measured in shed blood maintained at $37^{\circ} \mathrm{C}$ decreased on average $2.5 \mathrm{~mm} \mathrm{Hg}$ per min; this correction factor agrees with that suggested by Kelman and Nunn (20), and it was added to the determined Paos. Oxygen is consumed by the Clark $\mathrm{P}_{\mathrm{O}_{2}}$ electrode as well as by leukocytes during analysis (20). Therefore, a strip recording of oxygen tension with respect to time was obtained, and the slope was back extrapolated to the time of injection of blood into the cuvette for a more accurate estimate of oxygen tension.

Calculations. Arteriovenous oxygen difference $\left(\mathrm{A}-\mathrm{V} \mathrm{O}_{2}\right)$ in $\mathrm{ml} / 100 \mathrm{ml}$ of blood was determined from the product of the arteriovenous oxygen saturation difference in per cent, the blood hemoglobin concentration in $\mathrm{g} / 100 \mathrm{ml}$, and the normal oxygen capacity of hemoglobin $(1.34 \mathrm{ml} / \mathrm{g})$; the additional contribution of dissolved oxygen was determined by multiplying the Bunsen solubility coefficient $(0.003 \mathrm{ml}$ per $\mathrm{mm} \mathrm{Hg} / 100$ $\mathrm{ml}$ ) times the difference between arterial and mixed venous $\mathrm{P}_{\mathrm{O}_{2}}$. Alveolar-arterial oxygen tension differences (A-a $\mathrm{D}_{\mathrm{O}_{2}}$ ) were calculated from the determined $\mathrm{Pa}_{2}$ and the mean alveolar oxygen tension $\left(\mathrm{PA}_{\mathrm{A}_{2}}\right)$ determined from the alveolar air equation (21).

The fraction of true shunting was calculated from a modification of the Berggren formula $(22,23)$ :

$$
\dot{\mathrm{Q}}_{\mathrm{s}} / \dot{\mathrm{Q}}_{\mathrm{c}}=\frac{\left[\left(\mathrm{A}-\mathrm{a} \mathrm{D}_{2}\right)(0.003)+\mathrm{O}_{2} \operatorname{cap}\left(1-\mathrm{Sa}_{\mathrm{O}_{2}}\right)\right] 100 \% \mathrm{O}_{2}}{\mathrm{~A}-\mathrm{V} \mathrm{O}_{2}+\left[\left(\mathrm{A}-\mathrm{a} \mathrm{D}_{\mathrm{O}_{2}}\right)(0.003)+\mathrm{O}_{2} \operatorname{cap}\left(1-\mathrm{Sa}_{\mathrm{O}_{2}}\right)\right] 100 \% \mathrm{O}_{2}}
$$

where $\dot{Q}_{\mathrm{S}} / \dot{Q}_{\mathrm{c}}=$ the fraction of the cardiac output shunted, $\mathrm{O}_{2}$ cap $=$ the oxygen capacity of hemoglobin $/ 100 \mathrm{ml}$ blood, and $\mathrm{Sa}_{2}=$ the fraction of hemoglobin saturated with oxygen when the patient breathes $100 \%$ oxygen. When $\mathrm{Pa}_{2}$ is greater than $150 \mathrm{~mm} \mathrm{Hg}$, the term $\mathrm{O}_{2}$ cap $\left(1-\mathrm{Sa}_{O_{2}}\right)$ is neglected. It is apparent that maneuvers which reduce the $A-a D_{0}$ breathing $100 \%$ oxygen might do so either by reducing $\mathrm{A}-\mathrm{V}$ $\mathrm{O}_{2}$ difference (i.e., by increasing cardiac output) or by reducing the shunt (24). However, in three of the patients A-V $\mathrm{O}_{2}$ differences were measured both during spontaneous and assisted respiration, and the $\mathrm{A}-\mathrm{V} \quad \mathrm{O}_{2}$ difference increased slightly during assisted ventilation indicating that any decrease in $\mathrm{A}-\mathrm{a} \mathrm{D}_{\mathrm{O}_{2}}$ was caused by change in the shunt flow rather than change in cardiac output.

A calculation was made of the $\mathrm{A}-\mathrm{a} \mathrm{D}_{\mathrm{O}_{2}}$ breathing room air which could be explained by the shunt measured while the subject breathed $100 \%$ oxygen. These calculations were made assuming that the shunt caused the same oxygen deficit in

${ }^{1}$ Made by E. R. Squibb \& Sons, New York. arterial blood while breathing room air as while breathing $100 \%$ oxygen. Thus,

$$
\begin{aligned}
\left(\mathrm{PAO}_{2}-\right. & \left.\mathrm{PaO}_{2}\right)_{\text {air }} \\
& =\frac{(\text { numerator of eq } 1)-\mathrm{O}_{2} \mathrm{cap}\left(\mathrm{Sc}^{\prime} \mathrm{O}_{2}-\mathrm{Sa}_{2}\right)}{0.003}
\end{aligned}
$$

where $\left(\mathrm{PAO}_{2}-\mathrm{PaO}_{2}\right)_{\text {air }}=\mathrm{A}-\mathrm{a} \mathrm{D}_{2}$ breathing air. Knowing the alveolar oxygen tension breathing air $\left(\mathrm{PAO}_{2}\right)$ and assuming that the end-capillary $\mathrm{O}_{2}$ saturation $\left(\mathrm{Sc}^{\prime}{ }_{O_{2}}\right)$ is in equilibrium with the $\mathrm{PAO}_{2}$ at arterial $\mathrm{pH}$, the unique values of arterial $\mathrm{O}_{2}$ tension $\left(\mathrm{PaO}_{2}\right)$ and $\mathrm{O}_{2}$ saturation $\left(\mathrm{Sa}_{2}\right)$ are located on the oxyhemoglobin dissociation curve (25) which will satisfy equation 2 .

\section{RESULTS}

37 patients suspected of having acute pulmonary embolism had right heart catheterizations and pulmonary 
angiograms 0-19 (average 6) days after the first symptoms of the present episode of embolism. 21 of the $37 \mathrm{pa}-$ tients had positive angiograms and no other heart or lung disease, and form the basis of this report. Cardiac catheterization data of the patients are listed in Table I and arterial blood gas results in Table II.

Shunt-like effect. Each patient had one to four arterial blood gas determinations obtained 0-33 days after the first symptom of embolism. The measurements of A-a $\mathrm{D}_{\mathrm{O}_{2}}$ breathing $21 \% \mathrm{O}_{2}$ have been plotted against the A-a $\mathrm{Do}_{2}$ which could be accounted for entirely by the shunt measured while breathing $100 \% \mathrm{O}_{2}$ (Fig. 1); the points fall randomly around a line near the line of identity $(\mathrm{r}=0.80) ; P<0.005)$. The mean $\mathrm{A}-\mathrm{a} \mathrm{Do}_{2}$ measured with the patient breathing air exceeded the mean predicted $\mathrm{A}-\mathrm{a} \mathrm{Do}_{2}$ by only $5.5 \mathrm{~mm} \mathrm{Hg}$. Thus, true shunting accounted for most of the hypoxemia associated with acute pulmonary embolism in these patients with previously normal heart and lungs.

Shunting usually receded gradually over about a month following embolism (Fig. 2). However, hypoxemia that was severe persisted for only $2 \mathrm{hr}$ in one patient who, 2 days after the acute episode, showed $21 \%$ pulmonary vascular occlusion by angiography. The mean $\mathrm{A}-\mathrm{a} \mathrm{Do}_{2}$ breathing $100 \% \mathrm{O}_{2}$ the 1 st wk after diagnosis was not significantly different from the mean $\mathrm{A}-\mathrm{a} \mathrm{Do}_{2}$ the 2nd wk but was significantly different from the mean A-a $\mathrm{D}_{0_{2}} 3$ wk-6 months after the diagnosis $(P<0.05) .12$ patients had at least one repeat measurement, and paired $t$ test analysis of the first with the last $\mathrm{A}-\mathrm{a} \mathrm{Do}_{2}$ breathing $100 \% \quad \mathrm{O}_{2}$ showed a statistically significant reduction $(P<0.005)$.

13 patients had serial observations of $\mathrm{A}-\mathrm{a} \mathrm{D}_{\mathrm{o}_{2}}$ breathing room air and no recent surgical procedures. In seven of these, the $\mathrm{A}-\mathrm{a} \mathrm{Do}_{2}$ breathing room air increased at a later time when $\mathrm{A}-\mathrm{a} \mathrm{Do}_{2}$ breathing $100 \% \mathrm{O}_{2}$ had decreased. In one of the seven, the cause of the increasing $\mathrm{A}-\mathrm{a} \mathrm{Do}_{2}$ breathing room air was undoubtedly due to ventilation-perfusion mismatching. However, in the other six, the increased $\mathrm{A}-\mathrm{a} \mathrm{D}_{2}$ breathing room air was associated with some combination of increased $\mathrm{pH}$, increased $\mathrm{RQ}$, or decreased $\mathrm{Paco}-$ all of which exaggerate an $\mathrm{A}-\mathrm{a} \mathrm{D}_{\mathrm{o}_{2}}$ breathing room air that is due to a shunt-and the shunt could still explain the A-a $\mathrm{Doz}_{\text {o }}$ observed breathing room air.

The magnitude of the shunt did not correlate significantly with the per cent of vascular bed occluded $(r=0.07 ; P>0.30)$ nor with mean pulmonary artery pressure $(r=0.12 ; P>0.30)$.

When $100 \%$ oxygen was administered to these patients by intermittent positive pressure (IPPB) ${ }^{2}$ at pres-

\footnotetext{
${ }^{2}$ Abbreviations used in this paper: IPPB, intermittent positive pressure; FEV, forced expiratory volume; FVC, forced vital capacity.
}

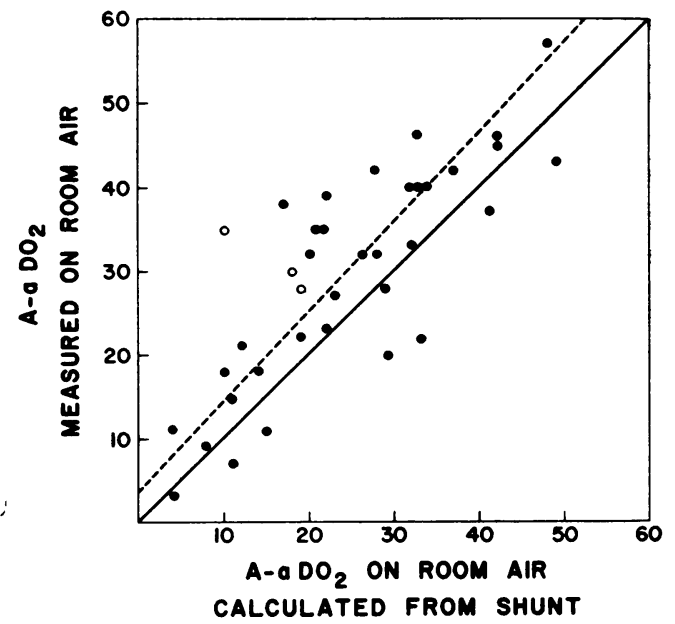

Figure 1 Alveolar-arterial oxygen tension differences during room air breathing are compared with the $\mathrm{A}-\mathrm{a} \mathrm{D}_{\mathrm{O}_{2}}$ that can be accounted for solely by the right-to-left shunt measured during $100 \%$ oxygen breathing. These data are from 18 of the patients before any surgical procedures and include one-four (average two) studies on each patient. The solid line is a line of identity, and the dashed line is the orthogonal regression line of best fit for the data. The open circles refer to measurements in three patients obtained before heparin administration.

sures of $20-40 \mathrm{~cm} \mathrm{H}_{2} \mathrm{O}$ causing tidal volumes of 1500 $4000 \mathrm{ml}$, two types of response were observed (Fig. 3). Patients with pulmonary infarction had a relatively small $\mathrm{A}-\mathrm{a} \mathrm{Do}_{2}$ breathing $100 \% \quad \mathrm{O}_{2}$ at normal tidal volumes, indicating a small shunt, and deep breathing caused little change. However, the maximum tidal volumes of these patients were only 60-69\% (average $65 \%$ ) of their predicted inspiratory capacities. Patients with emboli but no infarction had larger levels of A-a $\mathrm{Do}_{2}$ breathing $100 \% \mathrm{O}_{2}$ at normal tidal volumes, and the A-a $\mathrm{D}_{0_{2}}$ was decreased significantly by IPPB; in these patients tidal volumes achieved on IPPB were $80-111 \%$ (average $88 \%$ ) of the predicted inspiratory capacity. Five patients whose $\mathrm{A}-\mathrm{a} \mathrm{D}_{2}$ breathing $100 \% \mathrm{O}_{2}$ decreased during deep breathing continued to breathe $100 \%$ oxygen for 15 additional min after deep breathing, and the $\mathrm{A}-\mathrm{a} \mathrm{Do}_{\mathrm{o}_{2}}$ returned to the level observed before deep breathing. One patient performed voluntary inspiratory capacities while breathing room air. The A-a $\mathrm{Do}_{\mathrm{z}}$ breathing $21 \% \mathrm{O}_{2}$ was reduced from $29-20 \mathrm{~mm} \mathrm{Hg}$ and returned to $26 \mathrm{~mm} \mathrm{Hg}$ within $15 \mathrm{~min}$. Paired $t$ test analysis showed that deep breathing caused no significant change in $\mathrm{A}-\mathrm{a} \mathrm{D}_{0_{2}}$ breathing $100 \% \mathrm{O}_{2}$ in patients with infarction $(P>0.3)$ but caused a significant reduction in patients without infarction $(P<0.02)$.

10 of the 21 patients with positive pulmonary angiograms showed discoid atelectasis on roentgenograms of the chest. Only 1 of 10 patients with negative angiograms demonstrated discoid atelectasis, but no association be- 


\begin{tabular}{|c|c|c|c|c|c|c|c|}
\hline Patient & Age & Sex & $\begin{array}{l}\text { Angiogram } \\
\text { occlusion }\end{array}$ & $\begin{array}{c}\text { Mean } \\
\text { pulmonary } \\
\text { artery } \\
\text { pressure }\end{array}$ & $\begin{array}{l}\text { Wedge } \\
\text { pressure }\end{array}$ & $\begin{array}{c}\text { Right } \\
\text { ventricular } \\
\text { end-diastolic } \\
\text { pressure }\end{array}$ & Cardiac index \\
\hline & & & $\%$ & $m m H_{g}$ & $m m \mathrm{Hg}$ & $m m \mathrm{Hg}$ & liters $/ \min / m^{2}$ \\
\hline D. C. & 35 & $\mathbf{F}$ & 70 & 11 & 5 & 7 & $2.7^{*}$ \\
\hline S. M. & 31 & $\mathbf{F}$ & 3 & 11 & 6 & 5 & 4.0 \\
\hline M. S. & 59 & F & 68 & 18 & 9 & 6 & 3.0 \\
\hline H. Mc. & 27 & $\mathbf{F}$ & 358 & 25 & & & \\
\hline \multirow[t]{3}{*}{ L. M. } & 59 & $\mathbf{M}$ & 52 & 25 & & & $1.311(2.4)$ \\
\hline & & & 39 & 20 & 11 & & 2.5 \\
\hline & & & 3 & 13 & 5 & & 2.4 \\
\hline \multirow[t]{2}{*}{ N.S. } & 20 & $\mathbf{F}$ & $8 \S$ & 11 & 6 & 6 & 4.3 \\
\hline & & & 0 & 9 & & & $3.7^{*}$ \\
\hline \multirow[t]{2}{*}{ B. $\mathrm{T}$. } & 29 & $\mathbf{F}$ & 618 & 27 & 12 & 9 & 4.3 \\
\hline & & & 0 & 15 & & & \\
\hline \multirow[t]{2}{*}{ J. V. } & 65 & $\mathbf{M}$ & 35 & 22 & 5 & 5 & 2.8 \\
\hline & & & 3 & 16 & 8 & 8 & 2.6 \\
\hline \multirow[t]{2}{*}{ L. T. } & 56 & $\mathbf{F}$ & 808 & 24 & 7 & 15 & 2.3 \\
\hline & & & 11 & 15 & 6 & 8 & $3.4^{*}$ \\
\hline \multirow[t]{2}{*}{ W. W. } & 68 & $\mathbf{M}$ & $18 \S$ & 20 & 4 & 8 & 2.3 \\
\hline & & & 0 & 12 & & & \\
\hline J.P. & 44 & $\mathbf{F}$ & 14 & & & & \\
\hline \multirow[t]{3}{*}{ D. M. } & 25 & $\mathrm{~F}$ & $22 \S$ & 15 & 9 & 3 & $2.5^{*}$ \\
\hline & & & 18 & 15 & 8 & 8 & 2.3 \\
\hline & & & & 23 & & 12 & \\
\hline I. R. & 59 & $F$ & $5 \S$ & 10 & 3 & 4 & $2.6^{*}$ \\
\hline C. L. & 42 & $F$ & $29 \S$ & 23 & 5 & 2 & 3.7 \\
\hline \multirow[t]{2}{*}{ M. B. } & 48 & $\mathrm{~F}$ & 6 & 14 & 6 & 5 & 3.2 \\
\hline & & & 0 & 14 & 5 & 3 & 3.3 \\
\hline \multirow[t]{2}{*}{ B. L. } & 43 & $\mathbf{M}$ & $81 \S$ & 28 & 10 & 11 & 1.6 \\
\hline & & & 9 & 16 & 10 & 8 & 3.5 \\
\hline K. M. & 34 & $F$ & 268 & 14 & 5 & 5 & 3.3 \\
\hline L. H. & 43 & $\mathrm{~F}$ & 8 & 15 & & 7 & \\
\hline F. B. & 31 & $\mathbf{F}$ & 17 & 12 & 4 & 3 & 6.0 \\
\hline A. $\mathrm{R}$. & 53 & $\mathrm{~F}$ & 54 & 16 & 4 & 6 & 5.8 \\
\hline E. T. & 60 & $\mathbf{M}$ & 21 & 14 & 6 & 5 & 3.4 \\
\hline
\end{tabular}

* Fick cardiac output.

¥ Interval of time since the first symptom of the first episode of embolism studied.

$\S$ Pulmonary infarcts present on chest roentgenogram.

11 This Fick cardiac output is probably falsely low because of a technical error giving a $\dot{\mathrm{V}}_{\mathrm{O}_{2}}$ of $147 \mathrm{ml} / \mathrm{min}$. On six subsequent occasions $\dot{\mathrm{V}}_{\mathrm{O}_{2}}$ was $275 \pm 23 \mathrm{ml} / \mathrm{min}$. Using a $\dot{\mathrm{V}}_{\mathrm{O}_{2}}$ of $275 \mathrm{ml} / \mathrm{min}$ gives the cardiac index indicated in parentheses.

tween a positive pulmonary angiogram and the presence of discoid atelectasis could be demonstrated by chi square analysis $(P>0.1)$. Although there was a high incidence of pleuritic chest pain $(81 \%)$, there was not good correspondence between the severity of pain and the extent of shunting; some patients with severe pain had small shunts. In addition, the $\mathrm{A}-\mathrm{a} \mathrm{D}_{\mathrm{o}_{2}}$ breathing $100 \% \mathrm{O}_{2}$ was not significantly different in patients requiring no more than one dose of narcotics for pain compared with those requiring two or more doses of nar- cotics for the pain. The patients received on average 2.4 doses of narcotics (range 0-10 doses) before the first set of blood gases. After the first set of blood gases they received an average of 2.7 doses of narcotics (range 0-25 doses) over the next 2-3 wk.

Lung compliance. Specific dynamic compliance was performed on three patients with $\mathrm{A}-\mathrm{a} \mathrm{D}_{2}$ breathing $100 \% \mathrm{O}_{2}$ of $200-122 \mathrm{~mm} \mathrm{Hg}$. In two patients the A-a $\mathrm{D}_{2}$ breathing $100 \% \mathrm{O}_{2}$ was reduced $(61$ and $79 \%)$ and specific dynamic compliance increased $(17$ and $30 \%$ ) by 


\begin{tabular}{|c|c|c|c|c|c|c|}
\hline \multirow{2}{*}{$\begin{array}{c}\begin{array}{c}\text { Pulmonary } \\
\text { vascular } \\
\text { resistance }\end{array} \\
\text { dyne-cm } / \mathrm{sec}^{-5}\end{array}$} & \multicolumn{2}{|c|}{$\begin{array}{c}\text { Arteriovenous } \\
\text { oxygen } \\
\text { difference }\end{array}$} & $\begin{array}{c}\text { Time } \\
\text { since first } \\
\text { symptom } \neq\end{array}$ & $\begin{array}{c}\text { Time since } \\
\text { most recent } \\
\text { exacerbation } \\
\text { of symptoms }\end{array}$ & $\begin{array}{l}\text { Recurrent } \\
\text { emboli at } \\
\text { first study }\end{array}$ & $\begin{array}{l}\text { Recurrent } \\
\text { emboli since } \\
\text { last study }\end{array}$ \\
\hline & \multicolumn{2}{|c|}{$c c / 100 c c$} & days & $h r$ & & \\
\hline 100 & 3.3 & 3.4 & 2 & 24 & & \\
\hline 91 & 3.7 & 3.7 & 19 & 96 & & \\
\hline \multirow[t]{3}{*}{160} & & 4.7 & 14 & 168 & & \\
\hline & & & 14 & 24 & & \\
\hline & 5.1 & & 1 & 24 & $\mathrm{X}$ & \\
\hline 126 & 4.5 & 3.6 & 95 & & & \\
\hline 114 & & & 436 & & & $\mathrm{X}$ \\
\hline \multirow[t]{2}{*}{61} & 3.9 & 3.5 & 14 & 72 & & \\
\hline & 3.4 & 4.1 & 33 & & & \\
\hline \multirow[t]{2}{*}{148} & 3.7 & 3.6 & 15 & 24 & & \\
\hline & & & 39 & & & \\
\hline 278 & 5.0 & & 0 & 6 & $\mathrm{X}$ & \\
\hline 139 & 4.4 & 4.3 & 32 & & & \\
\hline 252 & 6.4 & 6.0 & 5 & 120 & & \\
\hline 99 & 3.8 & 4.0 & 29 & & & \\
\hline \multirow[t]{2}{*}{305} & 6.0 & 6.3 & 11 & 72 & & \\
\hline & & & 31 & 192 & & \\
\hline 117 & 3.8 & 3.8 & 7 & 96 & $\mathrm{X}$ & \\
\hline \multirow[t]{2}{*}{151} & 4.0 & 3.3 & 43 & & & $\mathrm{X}$ \\
\hline & 3.6 & & 163 & & & $\mathrm{X}$ \\
\hline 84 & 3.4 & 3.6 & 5 & 120 & & \\
\hline 169 & 3.9 & 3.4 & 19 & 48 & & \\
\hline 105 & 3.8 & 4.2 & 5 & 24 & & \\
\hline 174 & 3.6 & 3.7 & 18 & & & \\
\hline 480 & 5.1 & 3.8 & 1 & 6 & & \\
\hline 75 & 4.0 & 4.2 & 19 & 120 & & \\
\hline \multirow[t]{2}{*}{106} & 5.8 & & 5 & & & \\
\hline & & & 2 & 24 & & \\
\hline 65 & 3.1 & 4.1 & 5 & 72 & $\mathrm{X}$ & \\
\hline 96 & 3.3 & 3.6 & 8 & 96 & & \\
\hline 89 & 5.5 & 5.3 & 3 & 24 & & \\
\hline
\end{tabular}

deep breathing. In the third patient the $\mathrm{A}-\mathrm{a} \mathrm{Do}_{2}$ breathing $100 \% \mathrm{O}$, decreased less $(27 \%)$ during deep breathing than in the other two patients, and her specific dynamic compliance was not changed by deep breathing. The right-to-left shunt in this latter patient was at the atrial level as was demonstrated by a superior vena cava dye dilution curve.

Extrapulmonary shunts. In only 1 of 10 patients in whom superior vena cava (SVC) dye dilution curves were performed was right-to-left shunting demonstrated at the atrial level. In this patient (F. B.) pulmonary artery pressure was normal; mean right atrial pressure and mean pulmonary wedge pressure were both $4 \mathrm{~mm}$ $\mathrm{Hg}$, and the angiogram showed $17 \%$ occlusion of the pulmonary vascular bed.

Ventilation-perfusion-diffusing capacity mismatching. The average discrepancy between the measured $\mathrm{A}-\mathrm{a} \mathrm{Do}_{2}$ breathing air and that predicted from the shunt calculated with the $\mathrm{A}-\mathrm{a} \mathrm{D}_{\mathrm{O}_{2}}$ measured on $100 \%$ oxygen was only $5.5 \mathrm{~mm} \mathrm{Hg}$, a value easily explained by normal 
TABLE II

Arterial Blood Gas Studies during Hospitalization

\begin{tabular}{|c|c|c|c|c|c|c|c|c|c|c|c|c|c|}
\hline \multirow[b]{2}{*}{ Patient } & \multirow{2}{*}{$\begin{array}{l}\text { Hemo- } \\
\text { globin }\end{array}$} & \multirow[b]{2}{*}{$\mathrm{PAO}_{2}$} & \multicolumn{2}{|c|}{$\mathrm{PaO}_{2}$} & \multirow[b]{2}{*}{$\mathrm{PacO}_{2}$} & \multirow[b]{2}{*}{$\mathrm{pH}$} & \multirow{2}{*}{$\begin{array}{l}\text { Respi- } \\
\text { ratory } \\
\text { rate }\end{array}$} & \multirow{2}{*}{$\begin{array}{c}\text { Tidal } \\
\text { volume }\end{array}$} & \multirow[b]{2}{*}{$V_{D} / V_{T}$} & \multirow{2}{*}{$\begin{array}{c}\mathrm{PAo}_{2}-\mathrm{PaO}_{2} \\
\text { breathing } \\
100 \% \mathrm{O}_{2}\end{array}$} & \multirow{2}{*}{$\begin{array}{l}\text { Oxygen } \\
\text { consump- } \\
\text { tion }\end{array}$} & \multirow{2}{*}{$\begin{array}{l}\text { Respi- } \\
\text { ratory } \\
\text { quotient }\end{array}$} & \multirow{2}{*}{$\begin{array}{l}\text { Days } \\
\text { since } \\
\text { embolism }\end{array}$} \\
\hline & & & Meas & Pred & & & & & & & & & \\
\hline & $g m \%$ & $m m \mathrm{Hg}$ & \multicolumn{2}{|c|}{$m m H g$} & $m m \mathrm{Hg}$ & & & liters & & & $\mathrm{ml} / \mathrm{min}$ & & \\
\hline D. C. & 7.6 & 107 & 74 & 75 & 32 & 7.53 & 17 & 0.282 & 0.22 & 120 & 158 & 0.74 & 2 \\
\hline S. M. & 10.8 & 102 & 67 & & 35 & 7.47 & 17 & 0.398 & 0.41 & 200 & 148 & 0.86 & $19^{*}$ \\
\hline M. S. & 10.6 & & 74 & & 35 & 7.40 & & & & 140 & & & 14 \\
\hline H. Mc. & & & 80 & & 35 & 7.40 & & & & & & & 14 \\
\hline \multirow[t]{3}{*}{ L. M. } & 14.2 & 113 & 70 & 64 & 32 & 7.45 & 22 & 0.316 & 0.45 & 432 & 147 & 0.83 & 1 \\
\hline & 12.6 & 114 & 57 & 66 & 32 & 7.47 & 21 & 0.531 & 0.35 & 318 & 310 & 0.76 & 4 \\
\hline & 12.3 & 108 & 64 & 71 & 33 & 7.46 & 18 & 0.598 & 0.45 & 240 & 277 & 0.75 & 10 \\
\hline \multirow[t]{2}{*}{ N. S. } & 10.3 & 119 & 79 & 87 & 23 & 7.52 & 14 & 0.618 & 0.21 & 112 & 223 & 0.75 & 14 \\
\hline & 10.9 & 95 & 86 & 87 & 41 & 7.39 & 12 & 0.405 & 0.34 & 48 & 188 & 0.73 & 33 \\
\hline \multirow[t]{3}{*}{ B. $\mathrm{T}$. } & 11.6 & 96 & 75 & & 36 & 7.46 & 25 & 0.344 & 0.42 & & 273 & 0.66 & 15 \\
\hline & 11.2 & 108 & 81 & 85 & 36 & 7.44 & 17 & 0.478 & 0.31 & 140 & 249 & 0.85 & 18 \\
\hline & 11.7 & 101 & 83 & 91 & 40 & 7.44 & 14 & 0.483 & 0.32 & 58 & 237 & 0.81 & 22 \\
\hline \multirow[t]{4}{*}{ J.!V. } & 13.4 & 97 & 69 & 78 & 44 & 7.40 & 14 & 0.496 & 0.51 & 144 & 194 & 0.82 & 0 \\
\hline & 10.4 & 112 & 60 & & 28 & 7.54 & 28 & 0.661 & 0.52 & & 351 & 0.76 & $3^{*}$ \\
\hline & 9.4 & 110 & 72 & & 32 & 7.50 & 19 & 0.707 & 0.43 & 196 & 327 & 0.82 & $8^{*}$ \\
\hline & 10.2 & 106 & 76 & 85 & 35 & 7.52 & 17 & 0.678 & 0.40 & 96 & 271 & 0.83 & 32 \\
\hline L. T. & 11.6 & 99 & 67 & 79 & 32 & 7.44 & 17 & 0.272 & 0.42 & 134 & 139 & 0.61 & 5 \\
\hline & 9.8 & 95 & 62 & & 35 & 7.45 & 15 & 0.562 & 0.34 & 84 & 301 & 0.69 & $12^{*}$ \\
\hline w. W. & 17.0 & 83 & 68 & 72 & 42 & 7.39 & 20 & 0.230 & 0.60 & 140 & 119 & 0.60 & 11 \\
\hline & 15.4 & 91 & 80 & 76 & 37 & 7.44 & 19 & 0.441 & 0.55 & 127 & 235 & 0.61 & 15 \\
\hline & 15.3 & 84 & 77 & 73 & 44 & 7.44 & 21 & 0.469 & 0.53 & 103 & 257 & 0.65 & 22 \\
\hline J. P. & 7.8 & 97 & 75 & & 43 & 7.44 & 7 & 0.476 & 0.38 & & 118 & 0.80 & 8 \\
\hline & 7.6 & 94 & 83 & 79 & 35 & 7.41 & 9 & 0.634 & 0.38 & 62 & 218 & 0.77 & 15 \\
\hline D. M. & 9.2 & 102 & 80 & 83 & 34 & 7.46 & 15 & 0.316 & 0.32 & 91 & 155 & 0.72 & 7 \\
\hline & 9.3 & 101 & 80 & 89 & 37 & 7.46 & 12 & 0.649 & 0.47 & 60 & 218 & 0.75 & 12 \\
\hline I. R. & 6.6 & 98 & 58 & 66 & 33 & 7.49 & 16 & 0.260 & 0.38 & 153 & 140 & 0.58 & 5 \\
\hline & 5.9 & 118 & 72 & 76 & 27 & 7.54 & 16 & 0.583 & 0.29 & 113 & 215 & 0.89 & 11 \\
\hline C. L. & 10.7 & 99 & 62 & 58 & 34 & 7.48 & 13 & 0.613 & 0.37 & 311 & 290 & 0.64 & 19 \\
\hline & 10.7 & 93 & 70 & 71 & 39 & 7.44 & 12 & 0.593 & 0.41 & 147 & 255 & 0.69 & 23 \\
\hline & 10.7 & 106 & 88 & 92 & 32 & 7.46 & 13 & 0.566 & 0.22 & 70 & 278 & 0.75 & 30 \\
\hline M. B. & 13.0 & 103 & 63 & 69 & 39 & 7.44 & 20 & 0.371 & 0.47 & 213 & 181 & 0.85 & 5 \\
\hline & 13.0 & 94 & 66 & 65 & 35 & 7.48 & 23 & 0.303 & 0.43 & 248 & 241 & 0.58 & 9 \\
\hline & 11.4 & 98 & 66 & 70 & 36 & 7.44 & 23 & 0.289 & 0.57 & 196 & 149 & 0.68 & 18 \\
\hline & 10.4 & 127 & 82 & 85 & 24 & 7.58 & 22 & 0.629 & 0.34 & 112 & 214 & 1.10 & 32 \\
\hline B. L. & 15.2 & 99 & 64 & 89 & 38 & 7.43 & 42 & 0.214 & 0.65 & 94 & 155 & 0.72 & 1 \\
\hline & 12.9 & 112 & 73 & 90 & 30 & 7.48 & 23 & 0.472 & 0.32 & 122 & 283 & 0.82 & 4 \\
\hline & 13.2 & 78 & 75 & 74 & 42 & 7.42 & 11 & 0.524 & 0.40 & 44 & 275 & 0.56 & 13 \\
\hline & 12.8 & 117 & 102 & & 34 & 7.48 & 21 & 0.283 & 0.39 & & 106 & 1.14 & 19 \\
\hline K. M. & 13.6 & 98 & 60 & 81 & 38 & 7.48 & 23 & 0.385 & 0.36 & 139 & 305 & 0.72 & 5 \\
\hline & 13.5 & 117 & 71 & 84 & 32 & 7.54 & 19 & 0.602 & 0.36 & 144 & 222 & 1.13 & 8 \\
\hline & 11.4 & 123 & 81 & 95 & 28 & 7.55 & 15 & 0.863 & 0.21 & 79 & 267 & 1.15 & 13 \\
\hline L. H. & 14.6 & 110 & 78 & 84 & 31 & 7.46 & 12 & 0.638 & 0.29 & 159 & 224 & 0.81 & 4 \\
\hline F. B. & 12.9 & 107 & 72 & 86 & 36 & 7.47 & 17 & 0.565 & 0.38 & 122 & 268 & 0.86 & 5 \\
\hline A. $\mathbf{R}$. & 12.6 & 117 & 95 & 84 & 25 & 7.60 & 18 & 0.831 & 0.32 & 93 & 345 & 0.81 & 8 \\
\hline E. T. & 11.1 & 125 & 95 & 107 & 19 & 7.63 & 14 & 1.123 & 0.25 & 43 & 299 & 0.83 & 3 \\
\hline & 10.0 & 123 & 103 & 94 & 22 & 7.63 & 18 & 0.990 & 0.32 & 49 & 341 & 0.86 & 5 \\
\hline
\end{tabular}

* Blood gases may have been affected by complications of therapy in these studies. S. M. had had an iatrogenic pneumothorax requiring a chest tube on day 18, J. V. a vena caval ligation on day 1 , and L. T. an embolectomy on day 7 .

degrees of uneven ventilation-perfusion-diffusing capacity relationships.

11 patients had spirometry tracings during the 1 st wk after diagnosis at a time when increased A-a $\mathrm{Do}_{2}$ breathing $100 \% \mathrm{O}_{2}$ was still present. Eight had FEV 1.0 less than $85 \%$ of predicted, but seven of these also had restrictive ventilatory defects (FVC less than $85 \%$ of predicted). Only three of these eight had $\mathrm{FEV}_{1.0}$ less than $80 \%$ of $\mathrm{FVC}(57,78$, and $72 \%)$. In these three the $\mathrm{A}-\mathrm{a} \quad \mathrm{Do}_{2}$ breathing $100 \% \quad \mathrm{O}_{2}$ accounted for all of the
A-a $\mathrm{D}_{0_{2}}$ breathing air in two of the patients but explained only $75 \%$ of the $\mathrm{A}-\mathrm{a} \mathrm{Do}_{2}$ breathing air in the patient with the most severe obstructive ventilatory defect.

Two patients with moderate hypoxemia due to pulmonary embolism had determinations of slow space ventilation. One study was 5 days and another 32 days after the first symptom of embolism. Both patients demonstrated normal nitrogen clearance rates and a slowly ventilated compartment could not be clearly defined. 


\section{DISCUSSION}

Extent of embolic occlusion and reliability of its estimate. The estimated extent of embolic vascular occlusion varied from $3-81 \%$ of the pulmonary vascular bed and showed a good correlation with the reesting mean pulmonary artery pressure $(\mathrm{r}=0.66 ; P<0.01)$. Thus the study encompasses a wide range of severity. As pulmonary vascular bed becomes progressively occluded by emboli, the remaining fraction of the vascular bed must accept a progressively higher blood flow in order to sustain the normal cardiac output. It is a reasonable approximation to assume that if reflex pulmonary vasoconstriction does not occur after a pulmonary embolus the mean pulmonary artery pressure will rise no higher in response to the increment of blood flow through the remaining vascular bed than would be expected for a similar increment of pulmonary blood flow in a normal person from rest to exercise. Based upon this assumption the solid lines in Fig. 4 reflect the normal range of mean pulmonary artery pressures expected in our patients from the increments in resting flow through the remaining normal lung after taking into account the extent of occlusion. These ranges were obtained from composite data relating mean pulmonary artery pressure and cardiac output in normal persons at rest and exercise (26-35).

In only one instance was the pulmonary artery pressure higher than could be explained by simple mechanical obstruction; thus the data provide no support to the contention that generalized pulmonary vasoconstriction occurs after an embolus. In those instances where cardiac catheterization was repeated the fall of mean pulmonary

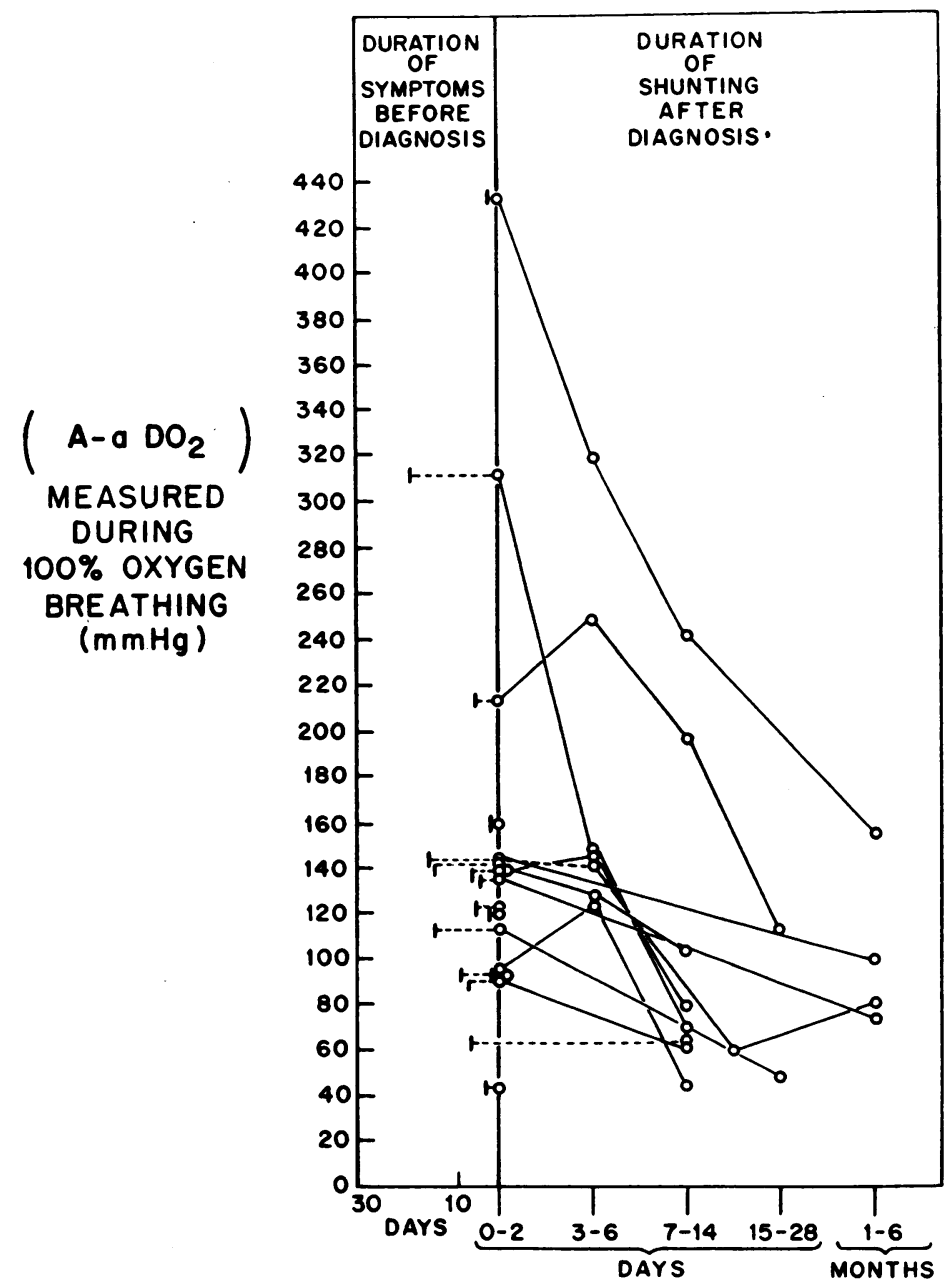

FIGURE 2 Duration of right-to-left shunting. The solid vertical line indicates the time of angiographic diagnosis of pulmonary embolism. The dashed lines to the left indicate the duration of symptoms before angiography, and the solid lines to the right indicate the $\mathrm{A}-\mathrm{a} \mathrm{D}_{\mathrm{O}_{2}}$ while breathing $100 \%$ oxygen as an estimate of shunting. 


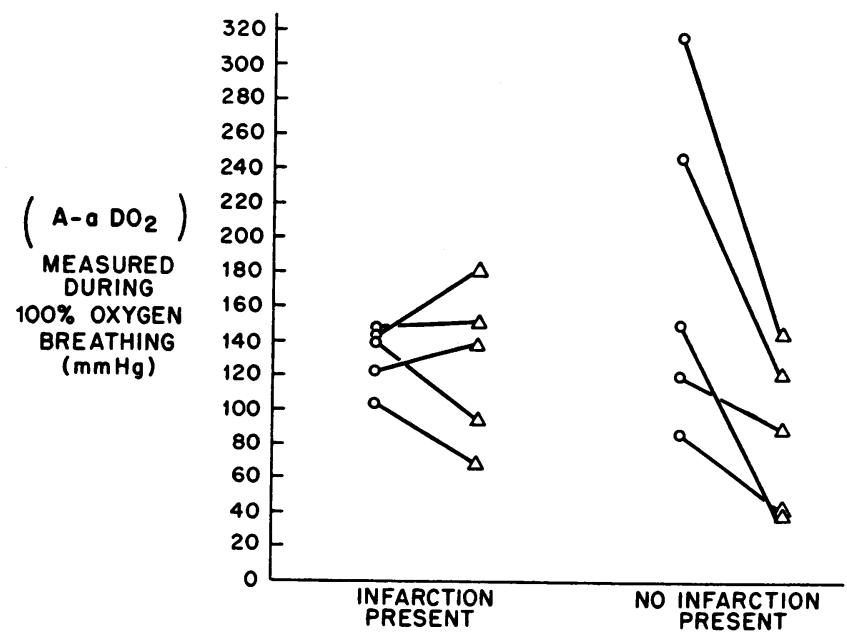

FIgURE 3 Changes in A-a $\mathrm{D}_{\mathrm{O}_{2}}$ during $100 \%$ oxygen breathing are shown before and after deep breathing. The open circles indicate measurements during normal tidal volumes, and the triangles indicate those during large tidal volumes. Tidal volumes of $60-69 \%$ of predicted inspiratory capacity were achieved in patients with pulmonary infarction, and tidal volumes of 80 $111 \%$ of predicted inspiratory capacity were possible in patients without lung infarction.

artery pressure seemed to accurately reflect the amount of angiographic clearing. This internal consistency of the results lends credence to the accuracy of the angiographic assessment of the extent of vascular occlusion. In only one instance was there a marked discrepancy between the estimated extent of occlusion and the level of resting mean pulmonary artery pressure (patient D. C.). The angiogram in this instance showed filling defects in both major pulmonary arteries without any sharp cutoffs; peripheral vasculature filled well and the scintillation scan showed no perfusion defect. The hemodynamic importance of the filling defects in this instance was obviously less than our arbitrary assessment of the angiogram indicated.

Shunts as the cause of hypoxemia. Among these patients who denied any prior lung disease, some degree of arterial hypoxia was almost always present within the first $3 \mathrm{wk}$ after a pulmonary embolus, although in no instance was the hypoxia profound. The lowest arterial $\mathrm{PO}_{2}$ breathing air was $57 \mathrm{~mm} \mathrm{Hg}$. The average A-a $\mathrm{Do}_{2}$ was $29.8 \mathrm{~mm} \mathrm{Hg}$ and $24.3 \mathrm{~mm} \mathrm{Hg}$ on average could be accounted for by the shunt measured while breathing $100 \%$ oxygen. Only $5.5 \mathrm{~mm} \mathrm{Hg}$ remain to be explained by mechanisms other than shunt. Since the unexplained $5.5 \mathrm{~mm} \mathrm{Hg}$ are no more than the normal $\mathrm{A}-\mathrm{a} \mathrm{Do}_{2}$ attributable to uneven ventilation-perfusion or uneven perfusion-diffusing capacity relationships in the lung, we must conclude that the major mechanism of the hypoxemia among our patients was right-to-left shunting of a fraction of the cardiac output. These results are in contrast to the findings of Kafer (8) who attributed most of the hypoxemia in pulmonary thromboembolic disease to ventilation-perfusion $(\dot{\mathrm{V}} / \dot{\mathrm{Q}})$ mismatching. However, most of his cases were studied in the subacute or chronic stage with a mean duration of symptoms of 29 months prior to study while our patients were studied within 19 days of the acute episode.

Most of our patients were receiving heparin by the time the first measurements were made. Considering only the measurements in which the $\mathrm{A}-\mathrm{a} \mathrm{Do}_{2}$ breathing air was above $20 \mathrm{~mm} \mathrm{Hg}$, there were 7 patients out of 16 in whom the shunt explains less than $70 \%$ of the measured A-a Do. (J. V., L. T., D. M., B. L., K. M., F. B., and E. T.). Three of these patients (J V., B. L., and E. T. noted as open circles in Fig. 1) were the only patients studied prior to heparin administration. In these three patients an average of $15 \mathrm{~mm} \mathrm{Hg}$ of the $\mathrm{A}-\mathrm{a} \mathrm{D}_{\mathrm{o}_{2}}$ remained unexplained by the shunt (approximately $50 \%$ of the total $\mathrm{A}-\mathrm{a} \mathrm{Do}_{2}$ ). Therefore, we have not ruled out the possibility that early heparin therapy eliminated some ventilation-perfusion mismatching which might have existed before heparin therapy. The tendency of heparin to relieve bronchospasm after pulmonary embolism has been reported by others (36). Furthermore, our data do not clearly exclude the possibility that much of the hypoxemia due to ventilation-perfusion disturbances could have spontaneously receded within hours after embolization before our initial measurements were made. 
There was mismatching of ventilation with perfusion in the form of wasted ventilation as indicated by an increased $V_{D} / V_{T}$ in 17 of 19 of our patients with the appropriate measurement. However, part of the explanation for increased $V_{D} / V_{T}$ is related to shallow breathing which increases the relative importance of anatomic dead space. It is difficult to quantitate whether wasted ventilation in one region leads to relative overperfusion in another region, but the rest of our data suggest that such an effect was minor in our patients.

The calculation of the fraction of the alveolar-arterial oxygen tension difference while breathing air that is due to true shunting is based on the assumption that the $\mathrm{A}-\mathrm{V} \mathrm{O}_{2}$ difference and the fraction of cardiac output shunted do not change from air to $100 \%$ oxygen breathing. These assumptions may not be invariably correct, but they are reasonable approximations which have been made by others $(24,37,38)$. We have experimental evidence that the $\mathrm{A}-\mathrm{V} \mathrm{O}_{2}$ difference did not change significantly from room air to $100 \%$ oxygen breathing during the cardiac catheterization study $(P$ $>0.6$ ). We had no independent way of estimating the fraction of cardiac output shunted under the two conditions, but it seems an unlikely source of systematic error since there were no other significant hemodynamic alterations between air and $100 \%$ oxygen breathing during cardiac catheterization.

Cause of right-to-left shunts. The lack of correlation between the mean pulmonary artery pressure and the per cent of the cardiac output shunted weighs against the opening of potential anatomical pathways for shunting which, though normally present, are usually not patent; this is a mechanism proposed but not proven by animal experimentation (39-42) and human autopsy studies $(43,44)$. Furthermore, our data indicate that intracardiac shunts occasionally do occur but were relatively infrequent among our subjects.

Both atelectasis (45) and temporary airway closure due to pneumoconstriction (46) or pulmonary edema (47) can cause right-to-left shunting that can be temporarily reversed by deep breathing. Indeed pulmonary edema has been suggested as one source of hypoxemia in animals following starch embolism $(3,48)$. In the present study the persistence of the shunting for several weeks after the embolus makes pulmonary edema unlikely as a cause. Furthermore, a significant obstructive ventilatory defect was uncommon suggesting that bronchoconstriction was not a major cause. The considerations above, along with the high incidence of discoid atelectasis observed after embolization on routine chest roentgenograms, favor atelectasis as the cause of the shunting. The fact that the shunts reappear so promptly after deep breathing and the fact that IPPB treatment does not seem to alter the natural, prolonged course of

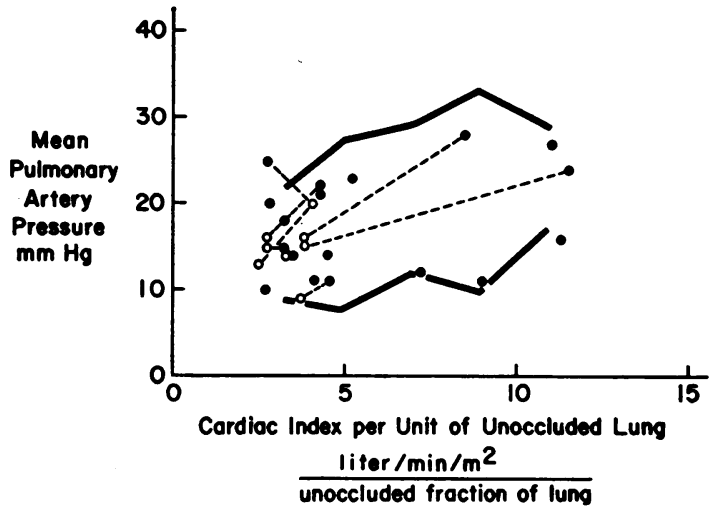

Figure 4 Mean pulmonary artery pressure is compared to cardiac index through unoccluded lung. The per cent of pulmonary vascular bed occluded was estimated from pulmonary angiograms. The closed circles represent the initial measurement. The open circles indicate repeat studies and are connected by straight lines to the corresponding data on the same patient obtained during earlier studies. In each instance a significant fall of mean pulmonary artery pressure occurred on the second study as the pulmonary arteries were recanalized. The solid lines represent the limits of mean pulmonary artery pressure expected from the increment in pulmonary blood flow through the remaining normal vascular bed; in only one instance was the pulmonary artery pressure greater than could be explained by the increase in cardiac output through unoccluded lung.

disappearance of these shunts make it apparent that the shunts are not simply a consequence of an altered pattern of breathing. The lungs seem mechanically predisposed to atelectasis. In order for the shunt-like effect to appear perfusion would have to be preserved or restored in the area of atelectasis. Possible explanations are that pulmonary emboli that only partially occlude flow cause alteration in surface-active properties enough to cause atelectasis, or that spontaneous fibrinolysis with clearing of some emboli occurs faster than restoration of surface active properties of the alveoli in that region. A similar process may explain shunting through infarcted areas that cannot be reversed by deep breathing-spontaneous fibrinolysis with recanalization may occur in the infarcted area where the alveoli cannot function either because of destruction or delayed clearance of red cells and debris. The duration of shunts agrees well with the time over which most of the clot lysis and arterial recanalization occurs.

\section{ACKNOWLEDGMENTS}

The authors wish to express their appreciation to Pat Wells for assistance with the manuscript and tables, and to Jim Harper, Brenda Beilby, and Janett Street for their technical assistance. Doctors D. S. Mierzwiak, N. P. S. Chawla, B. A. Khero, R. Robin, L. S. Cohen, and W. Shapiro generously assisted in performing part of the cardiac catheterization studies. 
This study was supported in part by a grant from the U. S. Public Health Service (HE 06296), and by a grant from the Dallas Heart Association.

\section{REFERENCES}

1. Wilson, J. E., III, W. R. Harrell, C. B. Mullins, E. R. Winga, R. L. Johnson, Jr., and A. K. Pierce. 1969. Hypoxemia in pulmonary embolism. J. Clin. Invest. 48: 89 a. (Abstr.)

2. Dalen, J. E., F. W. Haynes, F. G. Hoppin, Jr., G. L. Evans, P. Bhardwaj, and L. Dexter. 1967. Cardiovascular responses to experimental pulmonary embolism. Amer. J. Cardiol. 20: 3 .

3. Kabins, S. A., J. Fridman, J. Neustadt, G. Espinosa, and L. N. Katz. 1960. Mechanisms leading to lung edema in pulmonary embolization. Amer. J. Physiol. 198: 543.

4. Nemir, P., Jr., H. H. Stone, T. N. Mackrell, and H. R. Hawthorne. 1954. Studies on pulmonary embolism utilizing the method of controlled unilateral pulmonary artery occlusion. Surg. Forum 5: 210.

5. Comroe, J. H., Jr., B. Van Lingen, R. C. Stroud, and A. Roncoroni. 1953. Reflex and direct cardiopulmonary effects of 5-OH-trptamine (serotonin): their possible role in pulmonary embolism and coronary thrombosis. Amer. J. Physiol. 173: 379.

6. Jones, N. L., and J. F. Goodwin. 1965. Respiratory function in pulmonary thromboembolic disorders. Brit. Med. J. $1: 1089$.

7. Llamas, R., and E. W. Swenson. 1965. Diagnostic clues in pulmonary thrombo-embolism evaluated by angiographic and ventilation-blood flow studies. Thorax 20: 327.

8. Kafer, E. R. 1969. Respiratory function in pulmonary thromboembolic disease. Amer. J. Med. 47: 904.

9. Sasahara, A. A., and M. Stein, editors. 1965. Symposium on Pulmonary Embolic Disease, Boston, 1964. Pulmonary Embolic Disease; Proceedings. Grune and Stratton, New York. 257.

10. Sasahara, A. A., J. E. Cannilla, R. L. Morse, J. J. Sidd, and G. M. Tremblay. 1967. Clinical and physiologic studies in pulmonary thromboembolism. Amer. J. Cardiol. 20: 10 .

11. Sasahara, A. A., J. J. Sidd, G. Tremblay, and O. S. Leland, Jr. 1966. Cardiopulmonary consequences of acute pulmonary embolic disease. Progr. Cardiovasc. Dis. 9: 259.

12. Bass, H., J. E. Dalen, J. S. Banas, and L. Dexter. 1968. Effects of pulmonary embolus or infarction on lung function. Circulation 38: VI-38. (Abstr.)

13. Robin, E. D., C. E. Forkner, Jr., P. A. Bromberg, J. R. Croteau, and D. M. Travis. 1960. Alveolar gas exchange in clinical pulmonary embolism. N. Engl. J. Med. 262: 283.

14. Briscoe, W. A., and A. Cournand. 1959. Uneven ventilation of normal and diseased lungs studied by an opencircuit method. J. Appl. Physiol. 14: 284.

15. Briscoe, W. A. 1959. A method for dealing with data concerning uneven ventilation of the lung and its effects on blood gas transfer. J. Appl. Physiol. 14: 291.

16. Emmanuel, G., W. A. Briscoe, and A. Cournand. 1961. A method for the determination of the volume of air in the lungs: measurements in chronic pulmonary emphysema. J. Clin. Invest. 40: 329.

17. DuBois, A. B., S. Y. Botelho, and J. H. Comroe, Jr. 1956. A new method for measuring airway resistance in man using a body plethysmograph: values in normal subjects and in patients with respiratory disease. $J$. Clin. Invest. $35: 327$.

18. von Neergaard, K., and K. Wirz. 1927. Utber eine Methode zur Messung der Lungenelastizität am lebenden Menschen, insbesondere bein Emphysem. Z. Klin. Med. $105: 35$.

19. Mitchell, J. H., B. J. Sproule, and C. B. Chapman. 1958. The physiological meaning of the maximal oxygen intake test. J. Clin. Invest. 37: 538.

20. Kelman, G. R., and J. F. Nunn. 1966. Nomograms for correction of blood $\mathrm{P}_{\mathrm{O}_{2}}, \mathrm{P}_{\mathrm{CO}_{2}}, \mathrm{pH}$, and base excess for time and temperature. J. Appl. Physiol. 21: 1484.

21. Fenn, W. O., H. Rahn, and A. B. Otis. 1946. A theoretical study of the composition of the alveolar air at altitude. Amer. J. Physiol. 146: 637.

22. Berggren, S. M. 1942. The oxygen tension equilibrium between alveolar air and arterial blood. Acta Physiol. Scand. 4 (Suppl. 11) : 9.

23. McIlroy, M. B. 1965. Pulmonary shunts. Respiration, 2: (chapt. 65 , sect. 3 ).

24. Finley, T. N., C. Lenfant, P. Haab, J. Piiper, and $H$. Rahn. 1960. Venous admixture in the pulmonary circulation of anesthetized dogs. J. Appl. Physiol. $15: 418$.

25. Severinghaus, J. W. 1965. Blood gas concentrations. Respiration. 2: (chapt. 61, sect. 3).

26. Riley, R. L., A. Himmelstein, H. L. Motley, H. M Weiner, and A. Cournand. 1948. Studies of the pulmonary circulation at rest and during exercise in normal individuals and in patients with chronic pulmonary disease. Amer. J. Physiol. 152: 372.

27. Sancetta, S. M., and J. Kleinerman. 1957. Effect of mild, steady state exercise on total pulmonary resistance of normal subjects and those with isolated aortic valvular lesions. Amer. Heart J. 53: 404.

28. Dexter, L., J. L. Whittenberger, F. W. Haynes, W. T. Goodale, R. Gorlin, and C. G. Sawyer. 1951. Effect of exercise on circulatory dynamics of normal individuals. J. Appl. Physiol. 3: 439.

29. Fishman, A. P., H. W. Fritts, Jr., and A. Cournand. 1960. Effects of breathing carbon dioxide upon the pulmonary circulation. Circulation 22: 220

30. Freedman, M. E., G. L. Snider, P. Brostoff, S. Kimelblot, and L. N. Katz. 1955. Effects of training on response of cardiac output to muscular exercise in athletes. J. Appl. Physiol. 8: 37.

31. Hickam, J. B., and W. H. Cargill. 1948. Effect of exercise on cardiac output and pulmonary arterial pressure in normal persons and in patients with cardiovascular disease and pulmonary emphysema. J. Clin. Invest. 27: 10.

32. Donald, K. W., J. M. Bishop, G. Cumming, and O. L. Wade. 1955. The effect of exercise on the cardiac output and circulatory dynamics of normal subjects. Clin. Sci. (London). 14: 37.

33. Bevegård, S., A. Holmgren, and B. Jonsson. 1960. The effect of body position on the circulation at rest and during exercise, with special reference to the influence on the stroke volume. Acta Physiol. Scand. 49: 279.

34. Holmgren, A., B. Jonsson, and T. Sjöstrand. 1960. Circulatory data in normal subjects at rest and during exercise in recumbent position, with special reference to the stroke volume at different work intensities. Acta Physiol. Scand. 49: 343.

35. Slonim, N. B., A. Ravin, O. J. Balchum, and S. H. Dressler. 1954. The effect of mild exercise in the supine 
position on the pulmonary arterial pressure of five normal human subjects. J. Clin. Invest. 33: 1022.

36. Gurewich, V., D. Thomas, M. Stein, and S. Wessler. 1963. Bronchoconstriction in the presence of pulmonary embolism. Circulation 27 : 339.

37. Farhi, L. E., and H. Rahn. 1955. A theoretical analysis of the alveolar-arterial $\mathrm{O}_{2}$ difference with special reference to the distribution effect. J. Appl. Physiol. 7: 699.

38. Ayres, S. M., A. Criscitiello, and E. Grobovsky. 1964. Components of alveolar-arterial $\mathrm{O}_{2}$ difference in normal man. J. Appl. Physiol. 19: 43.

39. Rahn, H., R. C. Stroud, and C. E. Tobin. 1952. Visualization of arterio-venous shunts by cinefluorography in the lungs of normal dogs. Proc. Soc. Exp. Biol. Med. 80: 239.

40. Aviado, D. M. 1965. The Lung Circulation. Permagon Press, New York. 943.

41. Sirsi, M., and K. Bucher. 1953. Studies on arteriovenous anastomoses in the lungs. Experientia (Basel) 9: 217.

42. Prinzmetal, M., E. M. Ornitz, Jr., B. Simkin, and H. C. Bergman. 1948. Arterio-venous anastomoses in liver, spleen, and lungs. Amer. J. Physiol. 152: 48.
43. Tobin, C. E., and M. O. Zariquiey. 1950. Arteriovenous shunts in the human lung. Proc. Soc. Exp. Biol. Med. 75: 827.

44. Tobin, C. E. 1966. Arteriovenous shunts in the peripheral pulmonary circulation in the human lung. Thorax 21: 197.

45. Bendixen, H. H., J. Hedley-Whyte, and M. B. Laver. 1963. Impaired oxygenation in surgical patients during general anesthesia with controlled ventilation: a concept of atelectasis. N. Engl. J. Med. 269: 991.

46. Nadel, J. A., H. J. H. Colebatch, and C. R. Olsen. 1964. Location and mechanism of airway constriction after barium sulfate microembolism. J. Appl. Physiol. 19: 387.

47 Said, S. I., J. W. Longacher, Jr., R. K. Davis, C. M. Banerjee, W. M. Davis, and W. J. Wooddell. 1964. Pulmonary gas exchange during induction of pulmonary edema in anesthetized dogs. J. Appl. Physiol. 19: 403.

48. Swenson, E. W., R. Llamas, and J. Winkler. 1963. Hypoxemia in experimental pulmonary thromboembolism. Physiologist 6: 283. (Abstr.) 